\title{
The Oxford Pain Group League table of analgesic efficacy
}

\author{
Derek Richards \\ Director, Centre for Evidence-based Dentistry, Oxford, UK
}

Evidence-Based Dentistry (2004) 5, 22-23. doi:10.1038/sj.ebd.6400237

The two Canadian Collaboration on Clinical Practice Guidelines in Dentistry (CCCD) discussed in this issue of Evidence-Based Dentistry recommend the use of analgesics rather than antibiotic therapy for acute apical abscess where drainage cannot be achieved immediately, or in the treatment of acute apical periodontitis. Linked to this we have reproduced the Oxford League Table of Analgesic Efficacy: this is also freely available from the Oxford Pain Group internet site (www.jr2.ox.ac.uk/ bandolier/booth/painpag/index2.html) see Figure 1. Table 1 has been developed over many years by the Oxford Pain Research Group.

The information in the table has been derived from a large number of systematic reviews of randomised, doubleblind, single-dose studies in patients who had moderate to severe pain. Each of the reviews has the same outcome measure, at least $50 \%$ pain relief over $4-6 \mathrm{~h}$. The pain measurements were standardised, and have been validated. Numbers-needed-to-treat are calculated for the proportion of subjects who had at least $50 \%$ pain relief over 4-6h compared with placebo in rando- mised, double-blind, and single-dose studies in people with moderate to severe pain. Drugs were oral, unless specified, and doses are given in milligrams.

The relevance of this table to acute dental pain has been raised in this journal before in an editorial by Moore et al, ${ }^{1}$ when the author noted that dental pain is no different from other acute pain models.

1. Moore A. The evidence base in acute pain. Evid Based Dent 2000; 2:32-33.

2. Edwards JE, Oldman AD, Smith LA, et al. Oral aspirin in postoperative pain: a quantitative systematic review. Pain 2004; 107:86-90.

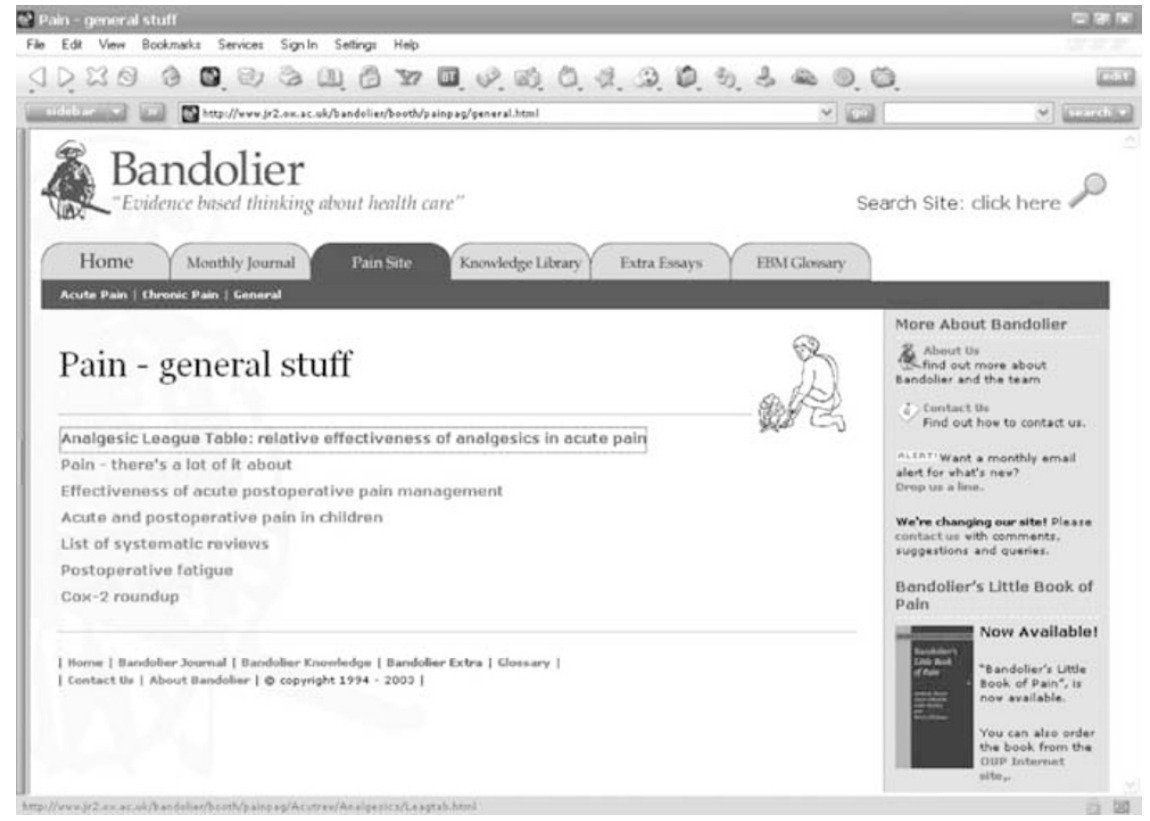

Figure 1. The Oxford Pain Group site. 
Table 1. The Oxford League table of analgesic efficacy.

\begin{tabular}{|c|c|c|c|c|c|}
\hline Analgesic and dose & $\begin{array}{c}\text { People in } \\
\text { comparison }(n)\end{array}$ & $\begin{array}{l}\text { Proportion with } \\
50 \% \text { pain relief }(\%)\end{array}$ & NNT & Lower $\mathrm{Cl}$ & Higher $\mathrm{Cl}$ \\
\hline Ibuprofen 800 & 76 & 100 & 1.6 & 1.3 & 2.2 \\
\hline Ketorolac 60 (intramuscular) & 116 & 56 & 1.8 & 1.5 & 2.3 \\
\hline Diclofenac 100 & 411 & 67 & 1.9 & 1.6 & 2.2 \\
\hline Piroxicam 40 & 30 & 80 & 1.9 & 1.2 & 4.3 \\
\hline Oxycodone IR 5 + paracetamol 500 & 150 & 60 & 2.2 & 1.7 & 3.2 \\
\hline Bromfenac 25 & 370 & 51 & 2.2 & 1.9 & 2.6 \\
\hline Rofecoxib 50 & 675 & 54 & 2.3 & 2.0 & 2.6 \\
\hline Diclofenac 50 & 738 & 63 & 2.3 & 2.0 & 2.7 \\
\hline Naproxen 440 & 257 & 50 & 2.3 & 2.0 & 2.9 \\
\hline Oxycodone IR 15 & 60 & 73 & 2.3 & 1.5 & 4.9 \\
\hline Bromfenac 100 & 95 & 62 & 2.6 & 1.8 & 4.9 \\
\hline Oxycodone IR 10 + paracetamol 650 & 315 & 66 & 2.6 & 2.0 & 3.5 \\
\hline Ketorolac 10 & 790 & 50 & 2.6 & 2.3 & 3.1 \\
\hline Ibuprofen 200 & 1414 & 45 & 2.7 & 2.5 & 3.1 \\
\hline Oxycodone IR 10 + paracetamol 1000 & 83 & 67 & 2.7 & 1.7 & 5.6 \\
\hline Piroxicam 20 & 280 & 63 & 2.7 & 2.1 & 3.8 \\
\hline Diclofenac 25 & 204 & 54 & 2.8 & 2.1 & 4.3 \\
\hline Dextropropoxyphene 130 & 50 & 40 & 2.8 & 1.8 & 6.5 \\
\hline Bromfenac 10 & 223 & 39 & 2.9 & 2.3 & 4.0 \\
\hline Pethidine 100 (intramuscular) & 364 & 54 & 2.9 & 2.3 & 3.9 \\
\hline Tramadol 150 & 561 & 48 & 2.9 & 2.4 & 3.6 \\
\hline Oxycodone IR 5 + paracetamol 1000 & 78 & 55 & 3.8 & 2.1 & 20.0 \\
\hline Paracetamol 600/650 + codeine 60 & 1123 & 42 & 4.2 & 3.4 & 5.3 \\
\hline Ibuprofen 100 & 396 & 31 & 4.3 & 3.2 & 6.3 \\
\hline $\begin{array}{l}\text { Paracetamol } 650+\text { dextropropoxyphene } \\
\text { ( } 65 \mathrm{mg} \text { hydrochloride or } 100 \mathrm{mg} \text { napsylate) }\end{array}$ & 963 & 38 & 4.4 & 3.5 & 5.6 \\
\hline Aspirin $600 / 650$ & 5061 & 38 & 4.4 & 4.0 & 4.9 \\
\hline Paracetamol 600/650 & 1886 & 38 & 4.6 & 3.9 & 5.5 \\
\hline Ibuprofen 50 & 316 & 31 & 4.7 & 3.3 & 7.9 \\
\hline Tramadol 100 & 882 & 30 & 4.8 & 3.8 & 6.1 \\
\hline Tramadol 75 & 563 & 32 & 5.3 & 3.9 & 8.2 \\
\hline Aspirin $650+$ codeine 60 & 598 & 25 & 5.3 & 4.1 & 7.4 \\
\hline Oxycodone IR 5 + paracetamol 325 & 149 & 24 & 5.5 & 3.4 & 14.0 \\
\hline Ketorolac 10 (intramuscular) & 142 & 48 & 5.7 & 3.0 & 53.0 \\
\hline Paracetamol $300+$ codeine 30 & 379 & 26 & 5.7 & 4.0 & 9.8 \\
\hline Bromfenac 5 & 138 & 20 & 7.1 & 3.9 & 28.0 \\
\hline Tramadol 50 & 770 & 19 & 8.3 & 6.0 & 13.0 \\
\hline Codeine 60 & 1305 & 15 & 16.7 & 11.0 & 48.0 \\
\hline Placebo & $>10000$ & 18 & NA & NA & NA \\
\hline
\end{tabular}

More information regarding the use of the table can be found at www.jr2.ox.ac.uk/bandolier/booth/painpag/Acutrev/Analgesics/Leagtab.html. NNT, Number-needed-to-treat; $\mathrm{Cl}$, confidence interval. 